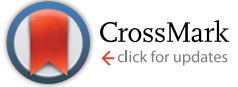

Cite this: RSC Adv., 2017, 7, 5002

Received 22nd October 2016 Accepted 9th December 2016 DOI: $10.1039 / c 6 r a 25671 f$

www.rsc.org/advances

\section{Elucidating the binding interaction of andrographolide with the plasma proteins: biophysical and computational approach $\uparrow$}

\author{
Daniel Pushparaju Yeggoni, ${ }^{a}$ Christian Kuehne, ${ }^{b}$ Aparna Rachamallu ${ }^{c}$ \\ and Rajagopal Subramanyam*a
}

\begin{abstract}
The present study focuses on the interactions of andrographolide (ANDR) with plasma proteins, human serum albumin (HSA), and $\alpha$-1-acid glycoprotein (AGP), and their biological importance. To understand the pharmacological role of ANDR, its anticancer activity was studied on a breast cancer cell line (MCF7); it showed a dose-dependent inhibition of growth, and its $I_{50}$ value was found to be $55 \mu \mathrm{M}$. Furthermore, to evaluate the binding mechanism of AGP and HSA with andrographolide, fluorescence emission quenching was observed as a static mechanism upon the binding of ANDR to plasma proteins. Additionally, active HSA sensor chip surfaces were prepared through an amine-coupling reaction protocol, and the equilibrium association constants for ANDR-HSA were then determined by surface plasmon resonance (SPR) analysis. The association constants of ANDR binding to HSA, obtained with fluorescence and SPR, were $K_{A(A G D)}=1.85 \pm 0.02 \times 10^{4} \mathrm{M}^{-1}$ and $3.1 \pm 0.04 \times 10^{3} \mathrm{M}^{-1}$, respectively. Similarly, the ANDR binding affinity with AGP was analyzed through fluorescence and SPR, and the calculated binding association values were $1.5 \pm 0.01 \times 10^{3} \mathrm{M}^{-1}$ and $1.3 \pm 0.04 \times 10^{3} \mathrm{M}^{-1}$, respectively. Molecular displacement and in silico docking shows that ANDR binds to subdomain IIB. Consequently, circular dichroism analysis showed that there is partial perturbation in the structure of HSA upon an increase in the concentration of ANDR. Moreover, molecular dynamics simulation revealed that the stability of the HSA-ANDR complexes reached an equilibration state at around 3000 ps, which clearly indicates the rigidity and stability of the HSA-ANDR complexes. Thus, our results provide evidence that both plasma proteins (HSA and AGP) can act as carrier proteins for ANDR.
\end{abstract}

\section{Introduction}

Human serum albumin (HSA) is the most abundant carrier protein and plays an important role in the transport and disposition of many endogenous and exogenous substances, such as metabolites, drugs, and other biologically active compounds, present in the blood. It consists of three structurally homologous domains, which assemble to form a heartshaped molecule, whereas each domain contains two subdomains. Albumin is known to bind and transport many ligands, including fatty acids, amino acids, hormones, cations and anions, and a variety of pharmaceuticals. It is suggested that the principal regions of ligand binding to HSA are located

aDepartment of Plant Sciences, School of Life Sciences, University of Hyderabad, Hyderabad 500046, India. E-mail: srgsl@uohyd.ernet.in; Fax: +91-40-23010120; Tel: $+91-40-23134572$

${ }^{b}$ Institute of Laboratory Medicine, Clinical Chemistry and Pathobiochemistry, ChariteUniversitätsmedizin Berlin, CVK, Augustenburger Platz 1, Berlin, 13353, Germany

'National Institute of Animal Biotechnology, Axis Clinicals Building, Miyapur, Hyderabad, 500049, India

$\dagger$ Electronic supplementary information (ESI) available. See DOI: $10.1039 / \mathrm{c} 6 \mathrm{ra} 25671 \mathrm{f}$ in the hydrophobic cavities in the subdomains IIA and IIIA, which are located at sites I and II, respectively. ${ }^{1,2}$ This is well characterized, as most drugs used in humans are bound to these two sites with an affinity constant of $10^{4}$ to $10^{6} \mathrm{M}^{-1} .^{3}$ It is widely accepted that the degree of affinity between a drug and HSA can dominate its distribution into the target tissue, affect its elimination from the body, and finally influence its therapeutic or toxic effects, biotransformation, and biodistribution. ${ }^{4}$ Besides, there is a testimony of conformational changes of the protein caused by its interaction with drugs, and these changes seem to affect the secondary and tertiary structure of protein. ${ }^{5}$ Therefore, investigating the interactions of a drug with HSA was very important, as this can provide useful information about drug actions and can be used as a model for elucidating the drug-protein complex.

Another important plasma protein, $\alpha$-1-acid glycoprotein (AGP), named as orosomucoid, comprised $2.5 \mathrm{mg} \mathrm{mL}^{-1}$ in human blood. This glycoprotein contains $45 \%$ carbohydrate, having a molecular weight of 41-43 kDa. Since it has a carbohydrate moiety, it gives $\mathrm{pH}$ 2.7-3.2 in aqueous solution. ${ }^{6,7}$ This hepatic protein, made up of 183 amino acids in a single polypeptide chain, is further attached to five asparaginyl-linked 
glycans. During acute phase response or inflammation, its glycosylation pattern varies and gives rise to a concentration 3-4 times more than normal; thus, AGP is referred to as a positive acute-phase protein. ${ }^{8}$ As well as its immunosuppressive properties, AGP has the ability to bind and transport several drug molecules, mostly lipophilic/cationic small molecules. As it has only one binding site, it can bind one molecule at a time, which makes it different from HSA. ${ }^{9}$

Furthermore, phytochemicals are known to bind serum proteins, and this is a good model for studying pharmacokinetics. Our group has extensively studied the interaction of various phytochemicals with serum proteins, and has observed that most of the phytochemicals bind strongly to HSA, ${ }^{\mathbf{1 0 - 1 2}}$ but only some of them bind to AGP. ${ }^{13,14}$ Andrographolide (ANDR) is a labdane diterpenoid, which is the main bioactive component of the medicinal plant Andrographis paniculata. ${ }^{15}$ ANDR has been reported to have a wide range of biological activities, such as anti-inflammatory, ${ }^{16}$ anti-allergic, ${ }^{17}$ anti-platelet aggregation, ${ }^{18}$ hepatoprotective, ${ }^{19}$ and anti-HIV properties. In biological systems, ANDR can interact with many inter- and intracellular constituents as a bipolar compound, thus inducing many biological responses. A recent study demonstrated that A. paniculata polysaccharides combined with ANDR can ease the recovery of diabetic nephropathy. ${ }^{20}$

Proteins are naturally-derived polymers that are advantageous in their biodegradability, low toxicity, non-antigenicity, high nutritional value, high stability, and binding capacity for various drugs, such as paclitaxel and ibuprofen. ${ }^{21-24}$ Plasma proteins are natural drug transporters, while nanocarriers are sometimes immunogenic, and their metabolite deposits may harm the body. Unlike plasma proteins, nanocarriers are artificial drug carriers, and when drug-loaded nanoparticles are injected into bodies, they cross epithelial barriers and circulate in the blood vessels before reaching the target site. There is a desire to conduct toxicology tests on the particles, since the different interactions of nanoparticles with fluids, cells, and tissues need to be considered, starting at the portal of entry and then via a range of possible pathways towards target organs. ${ }^{25}$ An alternative approach to designing nanocarriers is based on layer-by-layer (LbL) assembly technology. The assembly of LbL polymer capsules entails the sequential adsorption of interacting polymers onto sacrificial template particles, followed by template removal. The flexibility of this approach in terms of chemical nature and the properties of the polymers/template particles is limited only by the specific requirements related to therapeutic applications, such as biodegradability and biocompatibility. LbL technology confers the advantage of generating capsules with excellent structural stability, ${ }^{26}$ and allows the functionalization of nanoparticles smaller than $100 \mathrm{~nm}$, while restricting aggregation. The polymer layers are expected to act as a tool to control payload retention, degradation profiles, and drug release rates. ${ }^{27,28}$ However, the thickness of the polymer wall can affect cross-transport and therefore decrease the efficacy of active compounds encapsulated inside. In capsule-mediated delivery, diffusion may be difficult due to dense layers of fibrous tissue; drug amounts cannot be readily regulated.
Many agents are bound to serum proteins, especially serum albumin, which affects their pharmacologic and pharmacokinetic properties. Studies on the interaction of ANDR with HSA/ AGP will provide an insight into the chemical nature of the interaction of biomacromolecules with small molecules. This can be used as a model for elucidating ligand-protein complications. In addition, it is well known that the efficacy of many drugs in the body is based on the fact that drugs are carried by HSA/AGP; therefore, this experiment can supply some important methods to clinical research and provide a theoretical basis for designing new drugs. A variety of techniques are available for understanding the protein-ligand interaction in vitro; hence, an attempt has been made to use methods such as fluorescence quenching, surface plasmon resonance (SPR), molecular docking, and molecular dynamics simulations. Here, we emphasized particularly the mechanism and interactions of ANDR, a natural bioactive compound, with HSA/AGP, which include binding, protein-ligand stability, and conformation under physiological pH. Also, to understand the biological importance, we have used MCF-7 cell lines and studied the cell viability and cytotoxicity.

\section{Experimental}

\subsection{Materials and methods}

2.1.1 Preparation of stock solutions. Pure fat-free HSA (purchased from Sigma-Aldrich) was prepared with $1.5 \mathrm{mM}$ dissolved in 0.1 M phosphate buffer at $\mathrm{pH}$ 7.4. Andrographolide (bioactive compound) was purchased from Natural Remedies Pvt., Ltd, Bengaluru, India, with a purity of 95\%. Its stock solution $\left(1.0 \times 10^{3} \mathrm{M}^{-1}\right)$ was prepared in a $20: 80$ ethanol :water mixture, which is equivalent to $1: 4$. Through our previous reports, a solution containing $20 \%$ ethanol has no effect on the secondary structure of protein. ${ }^{29,47}$ The molecular weight of andrographolide is $350.4 \mathrm{Da}$. The optimum $\mathrm{pH}$ for HSA was set to be 7.4, as it has the maximum absorption at this $\mathrm{pH} .{ }^{29}$ Thus, for all the experiments, we have used $0.1 \mathrm{M}$ phosphate buffer at $\mathrm{pH} 7.4$ as a physiological buffer. All other chemicals are of analytical grade, purchased from SigmaAldrich. Research-grade CM5 sensor chips, 10× phosphate buffered saline (PBS), and an amine-coupling kit, consisting of $\mathrm{N}$-ethyl- $\mathrm{N}$-(3-dimethylaminopropyl)-carbodiimide (EDC), $\mathrm{N}$ hydroxysuccinimide (NHS), and ethanolamine hydrochloride $(\mathrm{pH} 8.5 ; 1 \mathrm{M})$, were all purchased from GE Healthcare BioSciences (Uppsala, Sweden).

2.1.2 Cell response assay. To carry out cell-response anticancer through MTT [3-(4,5-dimethylthiazol 2-yl)-2,5diphenyltetrazolium bromide] assay, MTT and all the cell lines, such as lung carcinoma (A549), human lung epithelial cells (BEAS-2B), prostate cancer (DU145), and breast cancer cells (MCF-7), used in the study were procured from ATCC (American Type Culture Collection). MCF-7 cells were taken in a 96-well plate, at a density of $5 \times 10^{5}$ cells, and sub-cultured and seeded. Then, the cells were treated with ANDR in a dose-dependent manner $(10 \mu \mathrm{M}$ to $100 \mu \mathrm{M})$ in a total volume of $100 \mu \mathrm{L}$. MTT crystals (20 $\mu \mathrm{L}$ in $5 \mathrm{mg} \mathrm{mL}{ }^{-1}$ PBS) were added to the plate and mixed after $48 \mathrm{~h}$ with $100 \mu \mathrm{L}$ of DMSO and incubated for $3 \mathrm{~h}$. 
Using a $\mu$-Quant Bio-tek Instrument, (microplate reader) the absorbance of the mixture was measured at $570 \mathrm{~nm}$. According to the result, the mean \pm standard error was calculated and plotted in a graph as the cell response (\%) vs. concentration $(\mu \mathrm{M})$, and $\mathrm{IC}_{50}$ values were calculated from the graph.

2.1.3 Fluorescence spectroscopy. The binding mechanisms of ANDR with macromolecules, HSA and AGP, were determined using fluorescence spectroscopy (Perkin-Elmer LS55 fluorescence spectrometer) with a $10 \mathrm{~mm}$ quartz cuvette. In the fluorescence emission measurement, fixed concentrations of HSA and AGP (0.001 mM), titrated with different concentrations of ANDR (0.001 $\mathrm{mM}$ to $0.009 \mathrm{mM})$ in $100 \mathrm{mM}$ phosphate saline buffer of $\mathrm{pH} 7.4$, were measured at $25{ }^{\circ} \mathrm{C}$. The bandwidths of both excitation and emission were fixed at $5 \mathrm{~nm}$, and a $1 \mathrm{~cm}$ quartz cuvette was used. For both HSA and AGP, the maximum excitation wavelength was kept at $285 \mathrm{~nm}$. The absorbance of ANDR at higher concentrations introduces the inner filter effect (IFE), which decreases the emission intensity of HSA and AGP, hence interfering with the quenching process. To eliminate the influence of the inner filter effect, which is a common inherent problem in many fluorimetric procedures, the fluorescence intensity was corrected using the following relationship. ${ }^{30}$

$$
F_{\text {cor }}=F_{\text {obs }} \times 10\left(A_{\text {exc }}+A_{\text {emi }}\right) / 2
$$

where $F_{\text {cor }}$ is the corrected fluorescence intensity, $A_{\text {exc }}$ and $A_{\text {emi }}$ represent the absorbance at the fluorescence excitation (285 $\mathrm{nm}$ ) and emission wavelengths (340 $\mathrm{nm}$ for AGP and $360 \mathrm{~nm}$ for HSA), and $F_{\text {obs }}$ is the observed fluorescence.

The emission spectra were collected in the wavelength range between $300 \mathrm{~nm}$ and $500 \mathrm{~nm}$ with a scan speed of $100 \mathrm{~nm}$ $\min ^{-1}$. This experiment was done thrice, keeping the number of accumulations to 3 times each to reduce noise. Thus, the fluorescence intensities used in the following sections are the corrected values.

2.1.4 Displacement experiment with site I (phenylbutazone) and II (ibuprofen). Site-selective experiments were performed at room temperature using two known site-specific probes; phenylbutazone for site I (IIA) and ibuprofen for site II (IIIA), with a fixed concentration of $0.001 \mathrm{mM}$, were mixed with HSA (0.001 $\mathrm{mM})$, and ANDR was added with increasing concentrations of $0.001 \mathrm{mM}$ to $0.009 \mathrm{mM}$. Here, other parameters were kept the same as for intrinsic fluorescence spectroscopy. The incubation times for the known markers were kept at $5 \mathrm{~min}$. The fluorescence intensity was recorded under the same experimental conditions as mentioned above. The fluorescence quenching data was analyzed using the SternVolmer equation. All fluorescence intensities were corrected for the absorption of excited and re-absorption of emitted light, and the IFE is corrected by using eqn (1).

2.1.5 SPR measurements. Experiments were carried out on a Biacore X100 device (GE Healthcare, Uppsala, Sweden). A carboxymethylated dextran chip (CM5-Chip, GE Healthcare, Uppsala, Sweden) was fully coupled on Fc2 with HSA or AGP (GE Healthcare), using an amine-coupling strategy (EDC/NHS) and HBS-EP (10 mM HEPES pH 7.4, $150 \mathrm{mM} \mathrm{NaCl,} 3$ mM EDTA, and $0.005 \% \mathrm{v} / \mathrm{v}$ surfactant $\mathrm{P} 20$ ) as a running buffer and sodium acetate $\mathrm{pH} 4.5-5.0$ as sample buffer for the ligand. At $\mathrm{pH}$ values above about 3.5, the carboxymethylated dextran on the sensor chip surface is negatively charged, and electrostatic attraction provides an efficient means for concentrating positively charged proteins on the surface. The $\mathrm{pH}$ of the protein solution should lie between 3.5 and the isoelectric point of the protein, so that the surface and the ligand carry opposite net charges. Covalent immobilization of proteins is best performed from solutions in $10 \mathrm{mM}$ buffer (e.g. sodium acetate) at $\mathrm{pH} 4-5.5$. These ready-touse buffers for protein immobilization are available from GE Healthcare. The response level reached $\sim 14650$ RU for HSA and $\sim 7803$ RU for AGP on Fc2. Non-binding Fc1 was mock-treated. Affinities were measured using a kinetic titration series (single cycle kinetics) at $25{ }^{\circ} \mathrm{C}$, in which five ascending concentrations of ANDR were injected consecutively for $120 \mathrm{~s}$ at $30 \mu \mathrm{L} \mathrm{min}{ }^{-1}$, followed by a dissociation time of $600 \mathrm{~s}$. Therefore, ANDR was diluted in the running buffer (PBS $+5 \%$ DMSO) at concentrations of $5 \mu \mathrm{M}, 25 \mu \mathrm{M}, 50 \mu \mathrm{M}, 200 \mu \mathrm{M}$, and $1000 \mu \mathrm{M}$. The signal of the untreated flow cell was subtracted from the binding signal. Additionally, for each run, later blank injections of running buffer only were also subtracted (double referencing). Sensorgrams were analyzed after solvent correction by plotting the analyte concentration against the binding signal at the end of the injection. The resulting isotherm was fitted to obtain the $K_{\mathrm{A}}$ values using the steady-state model.

2.1.5.1 Solvent refractive index correction. Since DMSO has a high refractive index, a solvent correction procedure was performed to account for the small variations in the DMSO percentage between samples and running buffer. ${ }^{31}$ PBS/DMSO mixtures ranging from $4.5 \%$ to $5.8 \%(\mathrm{v} / \mathrm{v})(\mathrm{pH} 7.4)$, were injected over the active and reference flow cells, and a calibration curve was obtained. This procedure is very important when working with small molecule-macromolecule interactions, especially when the ligand is immobilized at high density (5000 RU or more). In this experimental setup, the expected response has the same magnitude as, or a lower magnitude than, the signal arising from refractive index mismatches; therefore, including a correction protocol during the evaluation of the data becomes of great significance.

2.1.6 Circular dichroism spectroscopy. The changes in secondary elements, such as $\alpha$-helix, $\beta$-sheet, and random coil content, were monitored using CD spectroscopy. For recording the spectra of free HSA and the HSA-ANDR complex, a Jasco J815 spectropolarimeter was used at normal temperature $\left(25^{\circ} \mathrm{C}\right)$. Other parameters were followed as measurements ranged from 190-300 nm, including data pitch: $1 \mathrm{~nm}$, bandwidth: $2.00 \mathrm{~nm}$, scanning speed: $50 \mathrm{~nm} \min ^{-1}$, and accumulations: 3 . The final concentration of HSA was fixed at $0.001 \mathrm{mM}$, and ANDR was added in increasing concentrations from 0.001 to $0.009 \mathrm{mM}$. The spectra were analyzed for secondary structure determination with CDNN 2.1 software. The experiments were repeated thrice for ANDR, and each time approximate values were obtained.

2.1.7 Molecular docking. To determine the geometrically and energetically stable conformation upon binding of ANDR to HSA and AGP, molecular docking is the best method. Here, a predominant binding model of a protein (known structure) 
and ligand in 3D can be predicted. AutoDock 4.2.3 was employed to generate a docked conformation of ANDR with HSA and AGP; this uses a Genetic Algorithm (GA) and a Lamarckian Genetic Algorithm to generate conformations. Using this software, the binding free energies and binding sites of ANDR on HSA were investigated. The optimized 2D/3D structure of ANDR was built using Discovery Studio 3.5 software. Using Autodock, the water molecules were removed and non-polar hydrogen atoms were added. The crystal structure of HSA (PDB ID: 1AO6) was downloaded from the Brookhaven Protein Data Bank, and its functional groups were protonated in Autodock (4.2.3), which was chosen for docking studies, where water molecules were removed and hydrogen atoms were added. To define all binding sites, a grid box was generated with a spacing of $0.586 \AA$ and dimensions of $(126 \times 126 \times 126)$ points (.gpf file). The docking parameters were inserted as the number of GA runs: 30 , population size: 150 , the maximum number of generations: 27000 , the rate of genetic mutation: 0.02 , the rate of crossover: 0.8 , and GA crossover mode: 2 points. The output is selected as Lamarckian GA (.dpf file), which was implemented in Autodock (4.2.3) to conduct docking simulations. In the end, 30 possible conformers were obtained for HSA and ANDR binding. Among these, the one with the least binding free energy was considered for further analysis of binding mode and binding sites, as this was very close to experimental data. A similar protocol was followed (keeping the same parameters) to calculate the binding energy and efficiency of AGP (PDB ID: $3 \mathrm{KQO})$ and ANDR interactions.

2.1.8 Molecular dynamics simulations. After the ANDR is docked into the active site of HSA, MD simulations were performed using GROMACS v4.6.3, and this package was used to perform a simulation of the previous conformation (lowest binding energy docking conformation). The topology parameters were created by using the Dundee PRODRG2.5 server (beta). The entire simulation is performed on the basis of physiological conditions, as mentioned in the experimental method. The complex structure was immersed in a box of $7.335 \times 6.135 \times$ $8.119 \mathrm{~nm}^{3}$ dimensions with extended simple point charge (SPC) water molecules, and this system was neutralized by addition of $\mathrm{Na}^{+}$counter ions and 43626 solvent atoms. Therefore, the entire system consisted of HSA, ANDR, $\mathrm{Na}^{+}$of the counter-ions, and solvent atoms. The energy was minimized using the steepest descent method and the conjugate gradient method. Finally, the full system was subjected to $10 \mathrm{~ns}$ MD at a temperature of $300 \mathrm{~K}$ and 1 bar pressure. The atom coordinates were recorded every $0.5 \mathrm{ps}$ during the simulation. All restraints were then removed and each simulation was run for 10000 ps; simulation and analysis were performed on Linux cluster with 36 nodes (dual Xeon processor) at the Bioinformatics facility of the University of Hyderabad. ${ }^{32,33}$

\section{Results and discussion}

\subsection{Cytotoxicity study}

To reconfirm the importance of ANDR in cancer treatment, we performed a cell viability assay using MTT staining. ${ }^{34}$ Here, ANDR was used to induce apoptosis in MCF-7, a breast cancer cell line. The rate of apoptosis in the MCF-7 cell line is also dosedependent $(10,20,40,60,80$, and $100 \mu \mathrm{M})$, as the inhibition rate increases and the cell activity decreases in a concentrationdependent manner. Furthermore, we have calculated the $\mathrm{IC}_{50}$ value of ANDR to represent the cell viability, and this was found to be $55 \mu \mathrm{M}$ on MCF-7 cell lines (Fig. SI1†). Thus it can be concluded that ANDR has a strong anti-cancer activity, as well as other properties, such as anti-diabetic, anti-inflammatory, antiviral, antidyslipidemic, low-density lipoprotein oxidation, and antioxidant activity. Also, the biological effects of chemically synthesized ANDR and its analogs were well studied on several cancer cell lines, like lung carcinoma (A549), human lung epithelial cells (BEAS-2B), and prostate cancer (DU145), proving that ANDR is a potent drug possessing anticancer properties. $^{35}$ Therefore, our study on ANDR, which is very specific to MCF-7 cells, indicates that it can be used for the treatment of cancer. We also tried to understand the molecular level of the interaction of ANDR with plasma proteins.

\subsection{Binding analysis by fluorescence quenching mechanism}

In the present study, we have used fluorescence spectroscopy to unravel the binding mechanism or binding mode of ANDR to HSA or AGP. The fluorescence quenching of AGP and HSA is due to the intrinsic tryptophan residue, which is used as a tool to understand the interaction of ANDR with these proteins (Fig. 1A and B). While titrating ANDR with HSA, the fluorescence maximum intensity at $360 \mathrm{~nm}$ was decreased with the addition of ANDR. The major fluorescence of HSA is due to the presence of tryptophan-214 when excited at $280 \mathrm{~nm}$. This indicates that the ANDR binding site exists near to the tryptophan residue, which is located at the IIA subdomain of HSA.

In order to understand the fluorescence quenching mode (static (s) or dynamic (d)) of HSA and the AGP-ANDR complex, the quenching data were analyzed using the following equation:

$$
F_{0} / F=1+K_{\mathrm{q}} t_{0}[\mathrm{Q}]=1+K_{\mathrm{D}}[\mathrm{Q}]
$$

where $K_{\mathrm{D}}$ is the Stern-Volmer quenching constant $\left(K_{\mathrm{D}}=K_{\mathrm{q}} t_{0}\right)$, $K_{\mathrm{q}}$ is the bimolecular quenching rate constant, $t_{0}$ is the lifetime of the fluorophore in the absence of quencher, [Q] is the quencher concentration, and $F_{0}$ and $F$ are the fluorescence intensities in the presence and absence of the quencher. ${ }^{36-39}$ From the above equation, we have calculated the static quenching constant, $K_{\mathrm{q}}(\mathrm{s})_{(\mathrm{ANDR})}=5.0 \pm 0.03 \times 10^{13} \mathrm{M}^{-1} \mathrm{~s}^{-1}$ (Fig. SI $2 \dagger$ ). According to the standard procedures, if $K_{\mathrm{q}}$ is more than the maximum collisional quenching constant, then the static quenching is dominant. We found that the dynamic quenching constant is $2.06 \pm 0.03 \times 10^{10} \mathrm{M}^{-1} \mathrm{~s}^{-1} \cdot{ }^{32,40-43}$ Hence the binding of ANDR to HSA is 'static' as $K_{\mathrm{q}}(\mathrm{s})$ is very much greater than the colloidal constant. This indicates the formation of the fluorophore-quencher complex. The results demonstrated that HSA fluorescence quenching by ANDR may follow a static mechanism and was initiated by HSA-ANDR complex formation, rather than dynamic collision.

When small molecules are bound independently to a set of the equivalent sites on a macromolecule, and to maintain the 


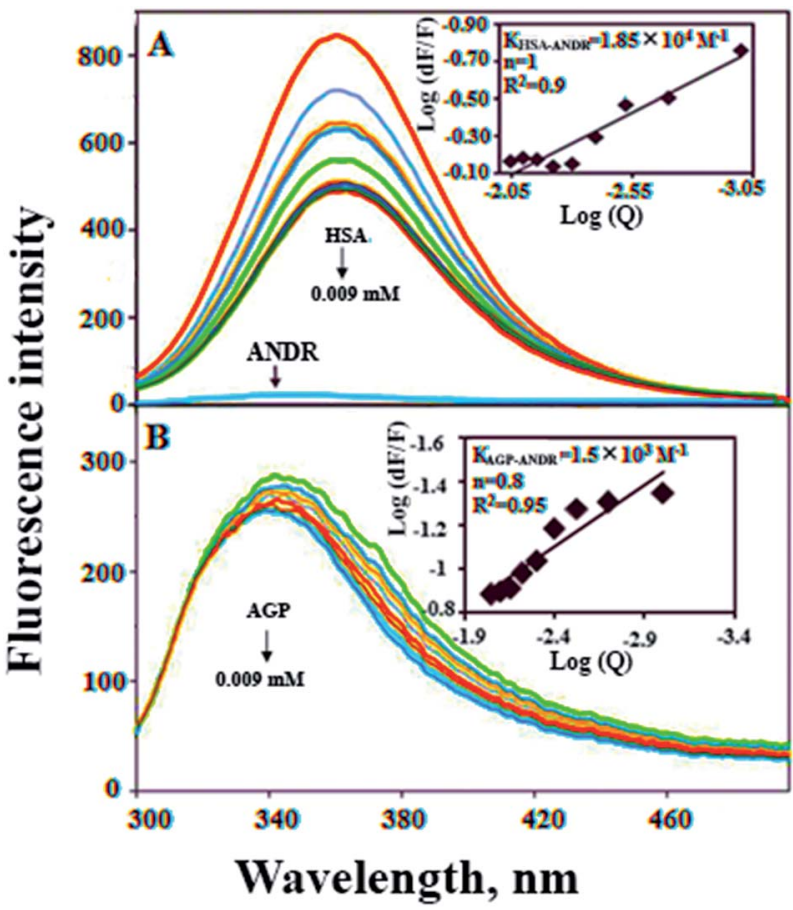

Fig. 1 Fluorescence emission spectroscopy of HSA and HSA-ANDR complex. (A) Free HSA $(0.001 \mathrm{mM})$ and free HSA with different concentrations of ANDR $(0.001,0.002,0.003,0.004,0.005,0.006$, $0.007,0.008$, and $0.009 \mathrm{mM}$ ) in $0.1 \mathrm{M}$ phosphate buffer, $\mathrm{pH} 7.4, \lambda_{\mathrm{ex}}=$ $285 \mathrm{~nm}$, temperature $=25^{\circ} \mathrm{C}$, inset: modified Stern-Volmer plot; plot of $\log (\mathrm{d} F / F)$ against $\log [\mathrm{Q}] \lambda_{\mathrm{ex}}=285 \mathrm{~nm}, \lambda_{\mathrm{ex}}=360 \mathrm{~nm}$. Fluorescence emission spectra of AGP-ANDR in $0.1 \mathrm{M}$ phosphate buffer with $\mathrm{pH} 7.4$, $\lambda_{\text {ex }}=285 \mathrm{~nm}$, and temperature $=25^{\circ} \mathrm{C}$. (B) Free AGP $(0.001 \mathrm{mM})$ and free AGP with different concentrations of ANDR at 0.001, 0.002, $0.003,0.004,0.005,0.006,0.007,0.008$, and $0.009 \mathrm{mM}$. Inset: plot of $\log (\mathrm{d} F / F)$ against $\log [\mathrm{Q}] . \lambda_{\mathrm{ex}}=285 \mathrm{~nm}$ and $\lambda_{\mathrm{em}}=340 \mathrm{~nm}$.

quenching in a linear relationship, the modified Stern-Volmer equation was used. .114,45 $^{1,45}$

$$
\log \left[\left(F_{0}-F\right) / F\right]=\log K_{\mathrm{s}}+n \log [\mathrm{Q}]
$$

where $n$ is the number of ligands binding to the protein, $K_{\mathrm{S}}$ is the binding constant, and $[\mathrm{Q}]$ is the drug concentration. The $\mathrm{d} F$ is $\left(F_{0}-F\right)$, where $F_{0}$ is the initial fluorescence of free HSA or AGP and $F$ is the fluorescence with different concentrations of ANDR.

We have plotted a graph of $\log (\mathrm{d} F / F)$ against $\log [\mathrm{Q}]$. From the graph, using the slope and the intercept values, the binding constant and the number of binding sites were calculated. We have found that the numbers of binding sites of ANDR to HSA and AGP are 1 and 0.8 for ANDR, supporting a one-to-one interaction between HSA and ANDR. Also, the binding constant (Fig. $1 \mathrm{~A}$ inset), $K_{\mathrm{ANDR}}=1.85 \pm 0.02 \times 10^{4} \mathrm{M}^{-1}$, indicates strong binding of ANDR with HSA. This data is well correlated with the data found in silico i.e. $1.2 \pm 0.03 \times 10^{4} \mathrm{M}^{-1}$. The same experimental procedures were followed at $37{ }^{\circ} \mathrm{C}$, where we found that on increasing the temperature, the fluorescence emission decreases, or in other words, the extent of lowering of fluorescence emission was higher at lower temperatures. For ANDR, the values of $K_{\mathrm{b}}$ and $n$ were calculated at $37^{\circ} \mathrm{C}$, and the observed values are $K_{\mathrm{b}}=1.2 \pm 0.03 \times 10^{4} \mathrm{M}^{-1}$ and $\Delta G=-5.5 \mathrm{kcal} \mathrm{M}^{-1}$ at $37^{\circ} \mathrm{C}$ (Table 1). Thus, the data show a slight decrease in $K_{\mathrm{b}}$ on increasing the temperature as a clear indication of static quenching. ${ }^{46}$ As we know, very few drug molecules bind efficiently with AGP; therefore, we have also calculated the binding constant, which was found to be $K_{\mathrm{ANDR}}=$ $1.5 \pm 0.03 \times 10^{3} \mathrm{M}^{-1}$; the in silico binding affinity of AGP is 7.7 $\pm 0.05 \times 10^{4} \mathrm{M}^{-1}$, and these are in the range of known FDA approved drug values $\left(10^{3}\right.$ to $\left.10^{6} \mathrm{M}^{-1}\right){ }^{3}$ Recently, we reported that few phytochemicals bind to AGP and HSA; ${ }^{13,14}$ from this point of view, we can state that due to the acute-phase protein, AGP, binding with ANDR, in chronic inflammation/cancer conditions, the ANDR can bind to overexpressed AGP. Thus, these results indicate that ANDR binds to both HSA and AGP. This reveals that there is only one binding site for ANDR for serum proteins, suggesting that both the proteins may be used as a carrier for ANDR in different disease conditions.

3.2.1 Calculation of free energy change. As the binding of HSA to the drugs is static, there will be four types of noncovalent forces, hydrogen bonding, van der Waals, hydrophobic, and electrostatic interactions, that stabilize their binding, which can be confirmed by calculation of the thermodynamic parameters. We know that the free energy change $\left(\Delta G^{\circ}\right)$ can be calculated using the following equation:

$$
\Delta G^{\circ}=-R T \ln K
$$

where $\Delta G^{\circ}$ is the free energy change, $R$ is the gas constant, $T$ is the absolute temperature, and $K$ is the binding constant. From the above equation, the free energy change for ANDR has been calculated as $-5.79 \pm 0.031 \mathrm{kcal} \mathrm{mol}^{-1}$ at $25^{\circ} \mathrm{C}$, when binding with HSA. Interestingly, this data also supports the in silico analysis of the HSA-ANDR complex, where $\Delta G_{\mathrm{ANDR}}^{\circ}=-5.6 \pm$ $0.031 \mathrm{kcal} \mathrm{mol}^{-1}$. Also, we have calculated the free energy change of binding to AGP and found this to be $-4.31 \pm 0.031$ $\mathrm{kcal} \mathrm{mol}^{-1}\left(\Delta G_{\mathrm{ANDR}}^{\circ}\right)$. Similar results were found in docking with AGP and ANDR, where $\Delta G_{\mathrm{ANDR}}^{\circ}=-6.67 \pm 0.21 \mathrm{kcal} \mathrm{mol}^{-1}$. Therefore, this data suggests that the binding between the protein and ligand can be stabilized by hydrophobic interaction and hydrogen bond formation. Similar results were recently published with other natural compounds (trans-feruloyl maslinic acid, betulinic acid, $\beta$-sitosterol, lupeol, $\mathrm{L}$-dopa, 7-hydroxycoumarin, trimethoxy flavone, embelin, and corilagin). ${ }^{12-14,29,32,47,48}$ The fluorescence quenching mechanism was found to be static, which is evident from the bimolecular quenching constant for ANDR of $5.27 \pm 0.02 \times 10^{13} \mathrm{M}^{-1} \mathrm{~s}^{-1}$. Quenching of the emission maximum at $340 \mathrm{~nm}$ of AGP was

Table 1 Binding parameters of ANDR interactions with HSA in $0.1 \mathrm{M}$ phosphate buffer, $\mathrm{pH} 7.4$, at different temperatures, obtained and calculated from fluorescence quenching results

\begin{tabular}{lll}
\hline$T(\mathrm{~K})$ & $K_{\mathrm{b}}\left(\times 10^{4} \mathrm{M}^{-1}\right)$ & $\Delta G\left(\mathrm{kcal} \mathrm{M}^{-1}\right)$ \\
\hline 298 & $1.85 \pm 0.01$ & -5.1 \\
310 & $1.22 \pm 0.03$ & -5.5
\end{tabular}


observed with ANDR. Interestingly, blue and red shifts were observed in the peaks of AGP-ANDR (Fig. 1B inset), whereas with HSA, there is no shift in the peaks of ANDR. This phenomenon might be due to the unfolding and refolding of the protein after binding. A possible explanation for the shift might be the differential exposure of tryptophan residues at various positions in the protein, which may be exposed differentially to the polar environment, leading to a shift in the emission maximum at $340 \mathrm{~nm}$. The binding constants with ANDR are 10 times less than that of HSA. ANDR binds with AGP in a $1: 1$ ratio, indicating that only one molecule is bound with AGP, and similarly with HSA. The negative free energies with all these concentrations of ANDR are less than the values obtained with HSA. From the binding constants and free energies of HSA and AGP, it is apparent that ANDR has a higher affinity to HSA than to AGP, signifying that the HSA-ANDR complex is more stable than the AGP-ANDR complex. There are several studies showing that AGP has moderate to high affinity $\left(10^{3}\right.$ to $\left.10^{6} \mathrm{M}^{-1}\right)$ with several drugs; ${ }^{49-51}$ some drugs showed equal binding with both HSA and AGP, ${ }^{52}$ and a few drugs, such as acetaminophen, phenobarbital, theophylline, and valproic acid, showed negligible binding to AGP. ${ }^{53}$ In our study, the ANDR compound showed less binding to AGP in comparison with HSA. These findings suggest that HSA plays a major role in binding; however, this should be verified in vivo in various pathological conditions where AGP expression is upregulated.

\subsection{Binding analysis from SPR data}

There are various techniques to understand molecular interactions; among these, fluorescence and SPR spectroscopy are widely used. Interactions between macromolecules and small molecules by fluorescence spectroscopy are based on the interaction between a quencher and a fluorophore. This allows fluorescence quenching, binding constants, and mechanisms of molecular interaction to be analyzed. SPR is an optical technique, which is based on the refractive index change near to a metal (Au) surface. The refractive index changes reflect the absorbance of analyte in solution onto the ligand HSA immobilized on the surface (Fig. 2). The advantage of SPR is that it can monitor the surface-constrained interaction in real time without the requirement for labeling. In this study, interaction between ANDR and HSA and AGP was studied by means of the above-mentioned techniques. The results showed that ANDR can be bound to HSA to form a complex. The association constants obtained from the fluorescence and SPR experiments were very close, namely, $1.85 \pm 0.02 \times 10^{4} \mathrm{M}^{-1}$ (fluorescence) and $3.1 \pm 0.04 \times 10^{3} \mathrm{M}^{-1}$ (SPR). As the interaction of ANDR with HSA is formed under flow conditions that usually cause shear stress, and takes place between an immobilized ligand and a mobile analyte, it might be slightly weaker than binding affinities derived from assays with both binding partners in solution without any shear stress forces, like fluorescence spectroscopy. Additionally, covalent coupling of HSA to the chip surface takes place randomly via primary amines in the protein. If these amines are situated next to the binding pocket for ANDR, interactions may be sterically hindered, reducing binding affinity in contrast to fluorescence spectroscopy, where no attachment is needed. However, we have also performed SPR by immobilizing AGP to investigate the interaction of ANDR with AGP under flow conditions. The binding affinities obtained from fluorescence and SPR spectroscopy were in good agreement with each other, at $1.5 \pm 0.03 \times 10^{3} \mathrm{M}^{-1}$ and $1.39 \pm 0.06 \times$ $10^{3} \mathrm{M}^{-1}$, respectively (Fig. SI $3 \dagger$ ). Steady-state analysis (weak and moderate interactions) data was initially extracted using Biacore X100 Control software, version 2.0.1. Hence, the binding constants obtained from the fluorescence and SPR are similar.

\subsection{Site selectivity binding studies of ANDR with HSA}

To determine the exact binding pocket of HSA upon interaction with ANDR, molecular displacement studies were carried out using site-specific markers for monitoring site I and site II of HSA. Here, we took two site-specific markers of HSA; phenylbutazone, which binds at IIA, and ibuprofen for IIIA, to determine the specific binding of ANDR with HSA through sitecompetitive displacement experiments. The concentration of HSA and those of site-specific markers are kept constant $(0.001$ $\mathrm{mM}$ ), and a decrease in fluorescence emission intensity was observed during titration of different concentrations of ANDR into HSA-phenylbutazone. The binding constant obtained was close to that of HSA-ANDR alone. From the displacement experiment, the binding constant decreased slightly in the

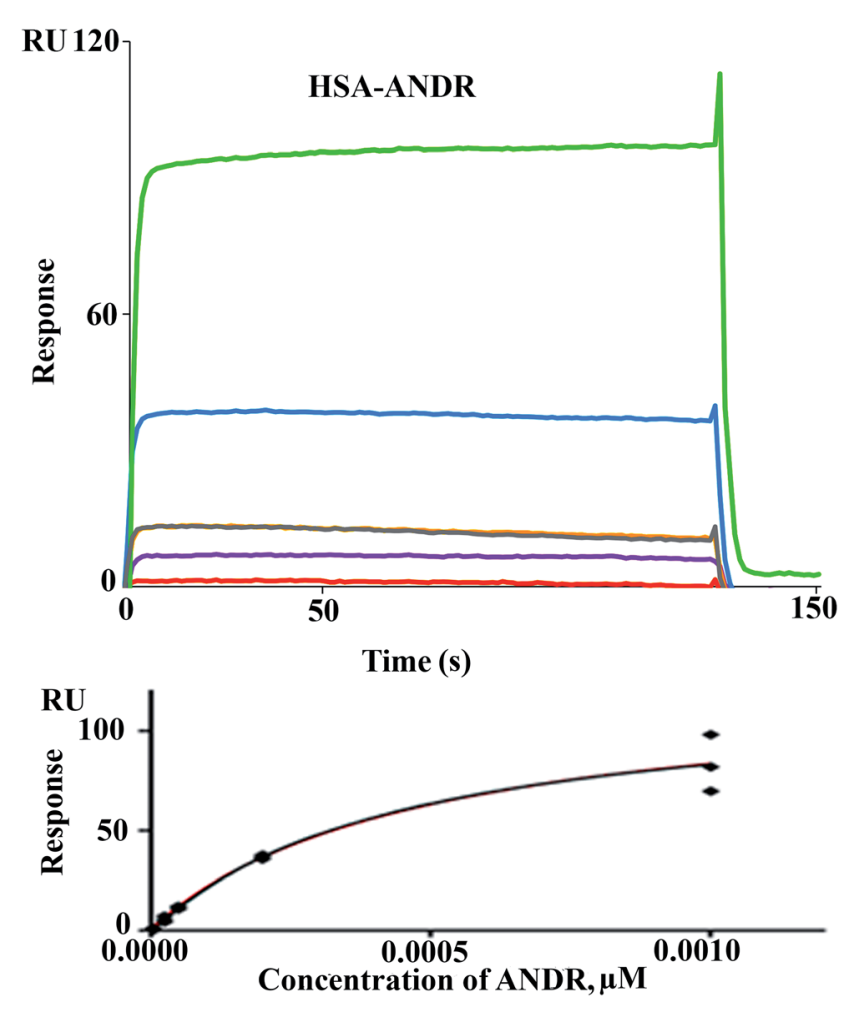

Fig. 2 Sensorgrams of binding of ANDR to HSA immobilized on CM 5 sensor chip (top) and respective $R_{\text {eq }}$ values fitted to the steady-state model, giving the final binding isotherm (bottom). Increasing concentrations of analyte are denoted by different colors: $5 \mu \mathrm{M}$ (orange), $25 \mu \mathrm{M}$ (purple), $50 \mu \mathrm{M}$ (light black and yellow), $200 \mu \mathrm{M}$ (blue), and $1000 \mu \mathrm{M}$ (green) for ANDR-HSA. 


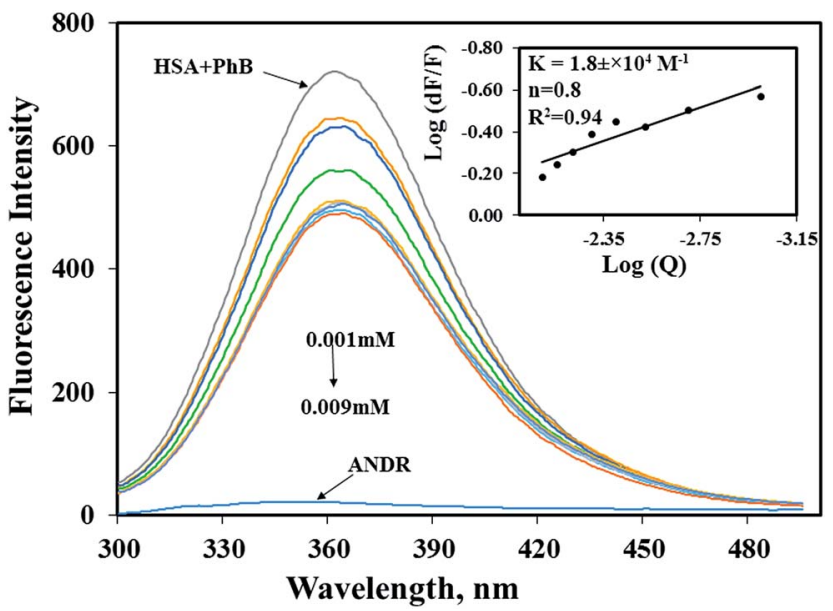

Fig. 3 Competitive binding of a ligand with HSA. Displacement of phenylbutazone from the HSA-phenylbutazone complex by ANDR. In the graph $\log d F / F v s . \log Q$ (inset) was plotted to calculate the binding constant and the number of binding sites.

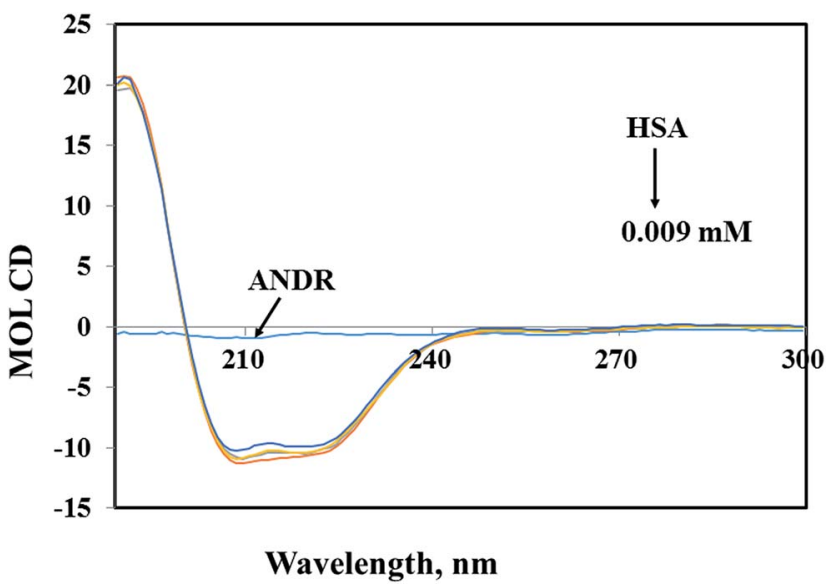

Fig. 4 Circular dichroism of the free HSA and HSA-ANDR complexes. The free HSA and HSA-ANDR complexes in aqueous solution had a protein concentration of $0.001 \mathrm{mM}$, and ANDR concentrations were $0.001,0.005$, and $0.009 \mathrm{mM}$.

presence of phenylbutazone, and was found to be $K_{\mathrm{ANDR}}=1.8 \pm$ $0.03 \times 10^{4} \mathrm{M}^{-1}$ (Fig. 3), which was very close to the intrinsic fluorescence data, which shows $K_{\mathrm{ANDR}}=1.85 \pm 0.02 \times 10^{4} \mathrm{M}^{-1}$. Therefore, competition was indicated between phenylbutazone and ANDR for binding site IIA of HSA. Our experimental data are consistent with the docking studies, which show ANDR binding to the IIA subdomain of HSA. However, there are no observed changes in the binding constants of other site-specific markers for the IIIA subdomain of HSA $\left(2.1 \pm 0.05 \times 10^{4} \mathrm{M}^{-1}\right)$, compared with the absence of the site-specific probe; thus, ANDR displaces only phenylbutazone from subdomain IIA. Therefore, from this study, we can assume that ANDR displaces the phenylbutazone molecule and forms a stable complex due to hydrophobic interactions at the IIA domain.

\subsection{Circular dichroism analysis}

Circular dichroism (CD) spectroscopy is a valuable technique for defining canonical secondary structures of proteins, based on empirically-defined spectroscopic signatures derived from proteins with known three-dimensional structures. CD (between $195 \mathrm{~nm}$ and $300 \mathrm{~nm}$ ) will yield secondary structural information. The chromophore being the peptide bond, the recorded spectrum will display specific signatures that can be related to certain peptide backbone arrangements. This plays an important role in understanding the secondary structural elements (percent of $\alpha$-helix, $\beta$-sheet, and random coil content) of proteins. A ligand binding to a globular protein may alter the secondary structure that results in the conformation..$^{54}$ In our study, the percentages of portions of secondary structural elements of the HSA-ANDR complex were evaluated. With the addition of an increased concentration of ANDR to HSA, the CDspectral behavior changes marginally (Fig. 4). Also, we have calculated the percentage of each of the secondary structural elements of HSA using CDNN 2.1 software. There were changes in the $\alpha$-helix, $\beta$-sheet, and random coil contents, as the ANDR concentration increased. The free HSA contains $56.0 \pm 2.5 \% \alpha$ helix, $25.6 \pm 0.62 \% \beta$-sheet, and $18.4 \pm 0.82 \%$ random coil (see Table 2). After gradual addition of ANDR from 0.001 to $0.009 \mathrm{mM}$, the $\alpha$-helical content decreased to $50.9 \pm 2.25 \%$, while the percentage of $\beta$-sheet and random coil content increased to $29.4 \pm 0.74 \%$ and $19.7 \pm 0.84 \%$, respectively. Similar results were observed, with a decreased $\alpha$-helical content and an increased $\beta$-sheet and random coil content, in earlier reports from our laboratory. ${ }^{\mathbf{1 1 - 1 3 , 2 9 , 5 5 - 5 7}}$ It is clearly evident that there was only a marginal decrease in the negative ellipticity in the region of far-UV CD, without any significant shift in peaks. The similarity between the CD spectral shapes of free HSA and HSA-ANDR suggested that the structure of HSA was also predominantly $\alpha$-helix (Table 2), indicating that the binding of the drug to HSA induced a slight decrease in the $\alpha$ helical structure content of the protein, with a minor increase in the $\beta$-sheets and random coils. The changes in the $\alpha$-helix of HSA indicated that the ANDR might have bound with the amino acid residues present in the HSA polypeptide chain and

Table 2 Percentage of the secondary structure of HSA and HSA-ANDR complex. Mean \pm SE $(n=3)$

\begin{tabular}{llcrr}
\hline Secondary structure (\%) & HSA & HSA-0.001 $(\mathrm{mM})$ & HSA-0.005 (mM) & HSA-0.009 (mM) \\
\hline Helix & $56.0 \pm 2.5$ & $56.4 \pm 2.5$ & $54.8 \pm 2.3$ & $26 \pm 0.62$ \\
Beta-turn & $25.6 \pm 0.62$ & $25 \pm 0.62$ & $19.2 \pm 0.83$ & $29.4 \pm 0.74$ \\
Random coil & $18.4 \pm 0.82$ & $18.6 \pm 0.82$ &
\end{tabular}


destroyed the hydrogen bonding networks. ${ }^{58}$ This might in turn have exerted a change in the secondary structure of the HSA. CD spectroscopy revealed conformational changes in the HSA, resulting from the change in the physiological environment of HSA after the addition of ANDR. Thus, ANDR created a variation in the local environment of HSA and changed its nature, indicating that the binding of ANDR to HSA induced a slight decrease in the $\alpha$-helical structure content of the protein, with a minor increase in the $\beta$-sheets and random coils. Although the binding of ANDR is greater $\left(1.85 \times 10^{4} \mathrm{M}^{-1}\right)$ to HSA, however, the protein conformation is not much observed due to orientational changes upon binding of ANDR to HSA.

\subsection{Binding studies from docking results}

To confirm the in vitro experimental data, molecular docking studies were carried out using Autodock (4.2.3) software and generated 30 different conformations with different binding conformations. Based on the lowest binding energy of the docking results, a conformer was taken and found to be $1.2 \pm$ $0.03 \times 10^{4} \mathrm{M}^{-1}$, and the free energy change was $-5.6 \pm 0.031$ kcal mol ${ }^{-1}$. Therefore, computationally calculated $K$ and $\Delta G^{\circ}$ values suggest that the binding of ANDR and HSA was similar to the results obtained with fluorescence data. These forces stabilize the docking conformation and are consistent with the binding mode obtained from the experimental results. It was also observed that ANDR binds at subdomain IIA of HSA in all of these 30 conformers. After analyzing the conformation of the complex, it was shown that ANDR binds in the hydrophobic pocket of subdomain IIA, and its binding was stabilized by the formation of $2 \mathrm{H}$-bonds between ANDR and GLN204 of HSA, with bond distances of 2.67 and $2.8 \AA$ (Fig. $5 \mathrm{~A}-\mathrm{C}$ ). Furthermore the microenvironment (around $4 \AA$ region) i.e. the active site of HSA, consisting of amino acid residues, Cys, Leu, His, Thr, Leu, Gly, Phe and Lys, etc. are in the vicinity of the ligand and conjugated with ANDR. From the binding site analysis, it is clearly evident that the ANDR-HSA complex is mainly stabilized by hydrophobic interactions. This supports the quenching of fluorescence, which is evident from the location of the ANDR, which is in close proximity to the tryptophan residue of HSA (Trp 214), suggesting the existence of hydrophobic interactions. The AGP and ANDR interaction was computationally calculated, and it was found that their interaction was stabilized in the ANDR-AGP complex by formation of $3 \mathrm{H}$-bonds with Pro 131, Lys 135 , and Thr 158 with distances of $2.1 \AA$ A $3.2 \AA$, and $3.12 \AA$, respectively (Fig. 6A-C). Furthermore, the binding constant $\left(K_{\mathrm{ANDR}}\right)$ is $7.7 \pm 0.05 \times 10^{4} \mathrm{M}^{-1}$ and the free energy change $\left(\Delta G_{\mathrm{ANDR}}^{\circ}\right)$ is $-6.67 \pm 0.21 \mathrm{kcal} \mathrm{mol}^{-1}$. These results support the experimental results $\left(K_{\mathrm{ANDR}}=1.5 \pm 0.03 \times 10^{3} \mathrm{M}^{-1}\right.$ and $\Delta G_{\mathrm{ANDR}}^{\circ}$ $=-4.31 \pm 0.031 \mathrm{kcal} \mathrm{mol}^{-1}$ ) found for the intrinsic fluorescence of the AGP and ANDR interaction. The interaction of ANDR with HSA is mainly stabilized by hydrophobic interactions, along with two hydrogen bonds, whereas with AGP, the main forces involved are three hydrogen bonds. ANDR shows robust binding to both HSA and AGP. Since AGP is an acutephase protein expressed in pathological conditions, binding of ANDR to the AGP warrants drug-binding studies.
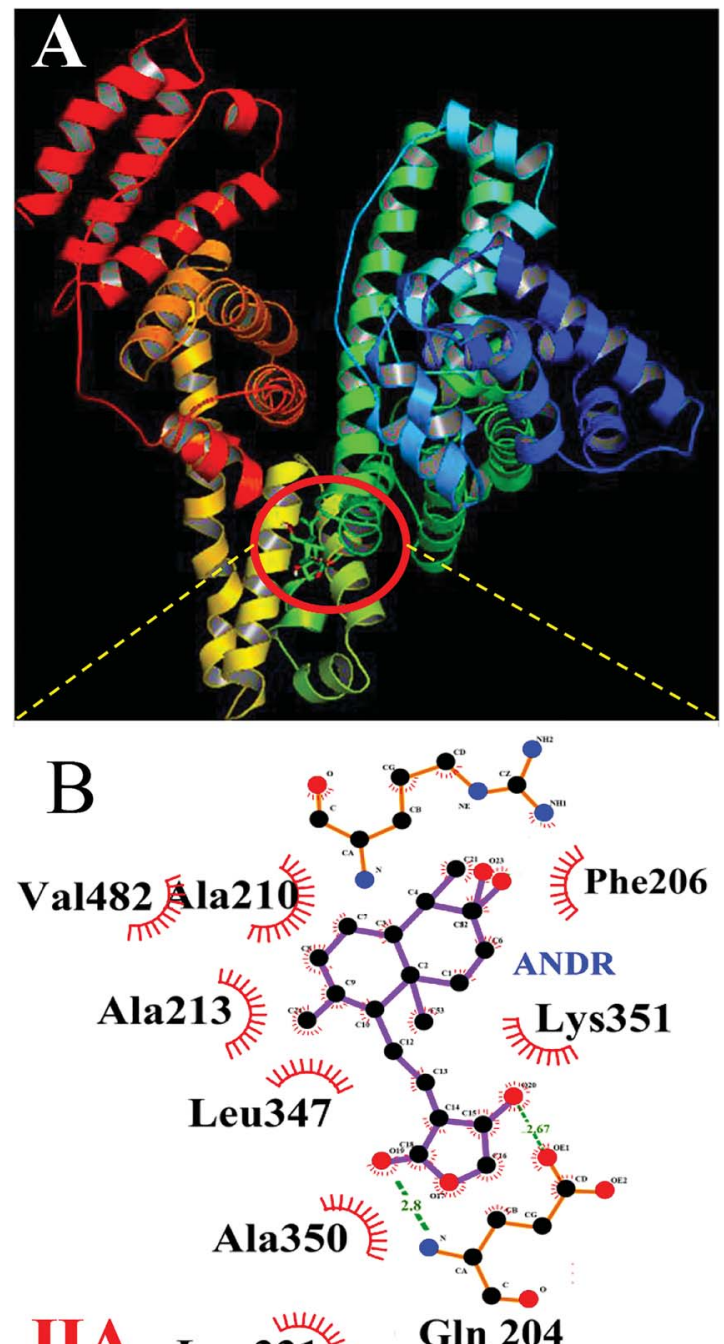

IIA Leu331 E Gln 204

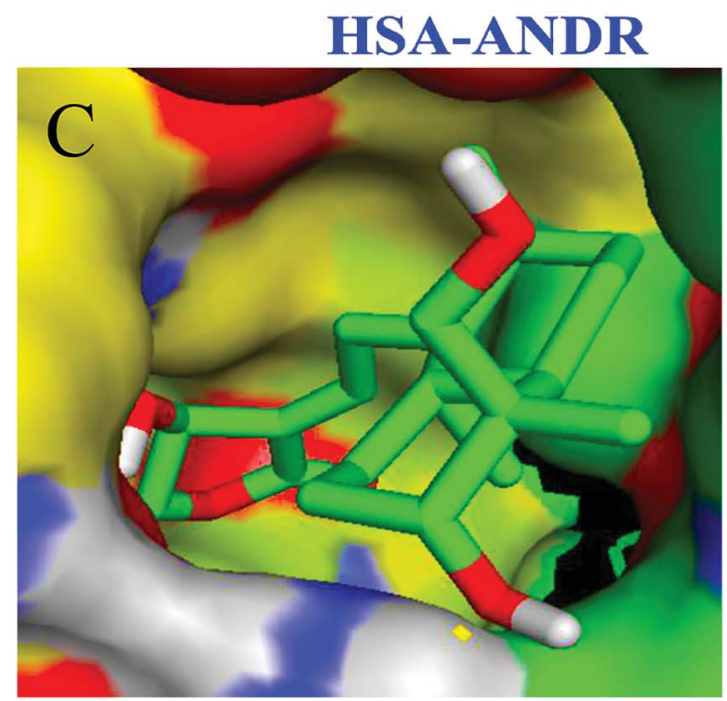

Fig. 5 Docking conformation of HSA-ANDR complex obtained from Autodock $v$ 4.2. (A) ANDR bound to IIA domain on HSA (protein represented in ribbon model and ligand represented in stick model). (B) Ligplot of ANDR binding site, showing two hydrogen bonds (Gln 204) and hydrophobic interactions of HSA with ANDR. (C) The hydrophobic pocket of the IIA domain surrounding the ANDR. 

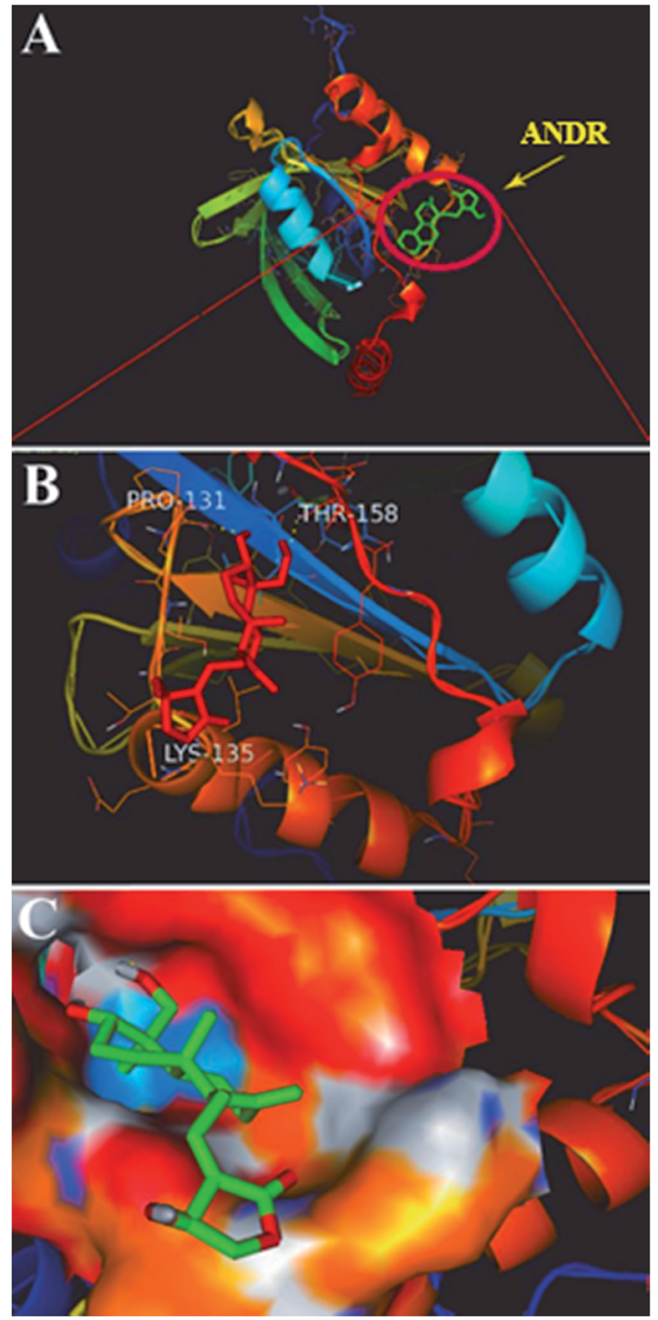

Fig. 6 Docking conformation of AGP-ANDR complex obtained from Autodock $\vee$ 4.2. (A) ANDR bound to AGP (protein represented in ribbon model and ligand represented in stick model). (B) Stereo view of ANDR bound to AGP (prepared by using Pymol v 1.5) in surrounding residues is represented as lines (three hydrogen bonds are with Pro 131, Lys 135, and Thr 158). (C) The binding pocket surrounding the ANDR.

\subsection{Rigidity of HSA-ANDR complexes observed from molecular dynamics simulation data (MD)}

In the above study, to investigate the binding affinity and secondary structural changes induced by ANDR, molecular dynamics simulations of HSA and the HSA-ANDR complex were performed and compared. So, to measure the structural properties for this study, we have used root mean square deviations (RMSD), radius of gyration $\left(R_{\mathrm{g}}\right)$, and root mean square fluctuation (RMSF), and the qualitative stability, rigidity, and conformational changes of the HSA were compared during the time of simulation.

Since HSA is an important protein in blood plasma, the stability, rigidity, and conformational changes of HSA with ligand molecules play a more important role than with AGP; hence, here we focused on HSA MD simulations with ANDR. Based on the agreement of experimental and computational
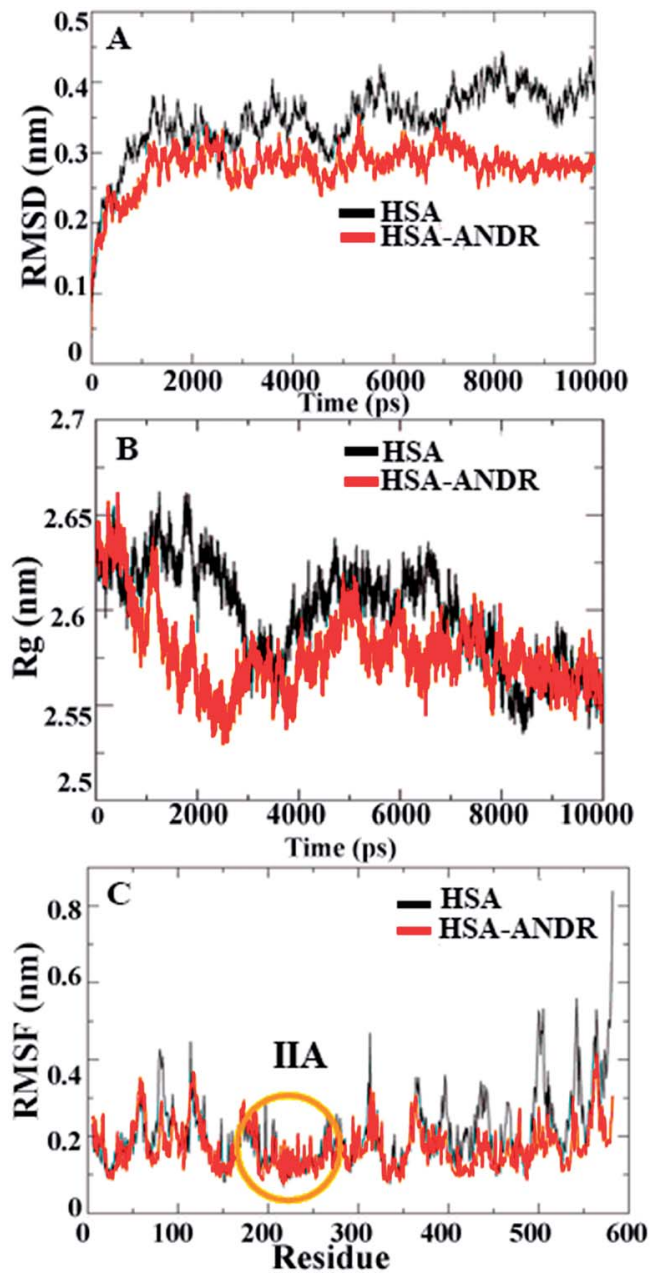

Fig. 7 Molecular dynamics simulation. (A) Time dependence of Root Mean Square Deviations (RMSD). $C_{\alpha}$ RMSD values for free HSA (black) and the HSA-ANDR (red) complex during a 10 ns MD simulation. (B) Time evolution of the radius of gyration $\left(R_{\mathrm{g}}\right)$. Radius of gyration $\left(R_{\mathrm{g}}\right)$ values during $10 \mathrm{~ns}$ of MD simulation of HSA (black) and HSA-ANDR (red) complex. (C) The RMSF values as a function of residue numbers. The RMSF values of free HSA (black) and HSA-ANDR (red) complex were plotted against residue numbers.

evidence, the lowest binding energy docked conformation of HSA-ANDR (bound to subdomain IIA) was chosen and used as a starting point for a $10 \mathrm{~ns}$ simulation. The RMSD values of atoms in free and ANDR-bound HSA were plotted from to 0-10 ns, as shown in Fig. 7, and the analysis indicated that the RMSD of both systems reaches equilibrium and oscillates around an average value after $3 \mathrm{~ns}$ simulation time. Fig. $7 \mathrm{~A}$ shows that the data point of fluctuation was determined as follows: for HSA, $0.40 \pm 0.035 \mathrm{~nm}$; HSA-ANDR, $0.29 \pm 0.056 \mathrm{~nm}$. The rigidity could be attributed to the unfolding of the protein, which is in agreement with the CD studies that showed minor changes in the secondary structural elements. Therefore, the changes are not due to destabilization and may be due to partial unfolding of HSA. So, we conclude that during MD simulation, there is no significant structural drift from the HSA structure. Recently, similar reports were documented from our lab related to protein stability. ${ }^{12-14,55}$ We also examined the time evolution of the 
radius of gyration $\left(R_{\mathrm{g}}\right)$ in the course of $10 \mathrm{~ns}$ of MD simulations of free HSA and the HSA-ANDR complex, and the results are shown in Fig. 7. The $R_{\mathrm{g}}$ values for the complex stabilized at about 4 ns and achieved equilibrium after 6 ns (Fig. 7B). Initially, the $R_{\mathrm{g}}$ values of free HSA and the complex with the drug were $2.64 \mathrm{~nm}$. The free HSA and HSA-ANDR were stabilized at $2.57 \pm 0.02 \mathrm{~nm}$ and $2.52 \pm 0.04 \mathrm{~nm}$. As expected, by reducing the radius of gyration and increasing the compaction rate in the binding to ANDR, all these findings align with those for lupeol and piperine complex formation with HSA. ${ }^{\mathbf{1 4 5 5}}$ So, based on the RMSD and $R_{\mathrm{g}}$ values obtained from our MD simulation data, it was concluded that HSA exhibits a slight structural change when combining with ANDR. Thus both experimental and computational results suggest slight conformational changes, probably at the binding region of the protein. Local protein mobility can be determined by RMSF values, which were plotted against residue numbers on a $10 \mathrm{~ns}$ trajectory. The RMSF plot shows the total amino acid fluctuation, which is the total polypeptide chain (Fig. 7C). The graph strongly indicates that the IIA subdomain for ANDR fluctuated less than others (IA, IB, IIB, IIIA, and IIIB). This data suggests that the drug molecule (ANDR) binds in the IIA subdomain and forms a rigid structure during MD simulation. The data clearly suggest that the ANDR binds specifically to the IIA subdomain, which supports the experimental evidence found from molecular displacement by site-specific markers. The rigidity of the binding sites from RMSF studies indicated that ANDR binds within subdomain IIA, which is in agreement with the docking studies. These results indicate that ANDR binds specifically to the respective domain, suggesting that binding sites are very specific for ANDR. Thus, during the simulation, the HSA-ANDR complexes are stable after 4 ns. Nonetheless, changes in the secondary structure were observed that many be due to partial unfolding of HSA. The unfolding of the protein structure may due to orientational changes upon binding of ANDR to HSA.

\section{Conclusion}

In the cell response study, the MCF-7 cell line of ANDR showed a concentration-dependent inhibitory effect; thus, it can be suggested that ANDR is a potent candidate against MCF-7 cell lines. Fluorescence studies of ANDR show quenching of the emission spectra of HSA and AGP proteins. Furthermore, the binding efficiency was measured by two methods, i.e. fluorescence emission and SPR, for ANDR with HSA. The binding constants from both techniques were found to be close. Furthermore, CD data reveals that the $\alpha$-helical content is increased as compared with the $\beta$-sheet and random coil contents, indicating that partial changes in the secondary structural elements are shown in HSA. Additionally, site-specific marker experiments show ANDR competing with phenylbutazone, thereby replacing it for the binding site of subdomain IIA; this supports the molecular docking results, further indicating the formation of a hydrogenbond-stabilized HSA-ANDR complex. Furthermore, MDS studies indicated that the docked conformer is stable. In a timedependent simulation study, ANDR binds at the IIA subdomain within 4000 ps and is stabilized. Hence, our observation allowed us to understand the molecular level interaction and characterization of ANDR with plasma proteins and thus it can be further utilized as a therapeutic agent.

\section{References}

1 D. Carter, X.-M. He, S. H. Munson, P. D. Twigg, K. M. Gernert, M. B. Broom and T. Y. Miller, Science, 1989, 244, 1195-1198.

2 X. M. He and D. C. Carter, Nature, 1992, 358, 209-215.

3 A. Varshney, P. Sen, E. Ahmad, M. Rehan, N. Subbarao and R. H. Khan, Chirality, 2010, 22, 77-87.

4 J. Flarakos, K. L. Morand and P. Vouros, Anal. Chem., 2005, 77, 1345-1353.

5 F. Ding, W. Liu, N. Li, L. Zhang and Y. Sun, J. Mol. Struct., 2010, 975, 256-264.

6 K. Schmid, R. B. Nimberg, A. Kimura, H. Yamaguchi and J. Binette, Biochim. Biophys. Acta, 1977, 492, 291-302.

7 T. Fournier, N. Medjoubi-N and D. Porquet, Biochim. Biophys. Acta, Protein Struct. Mol. Enzymol., 2000, 1482, 157-171.

8 J. M. Kremer, J. Wilting and L. Janssen, Pharmacol. Rev., 1988, 40, 1-47.

9 Z. Huang and T. Ung, Curr. Drug Metab., 2013, 14, 226-238.

10 D. P. R. Yeggoni, M. M. Darla, C. S. Reddy and R. Subramanyam, J. Biomol. Struct. Dyn., 2015, 34, 1-46.

11 D. P. Yeggoni and R. Subramanyam, Mol. Biosyst., 2014, 10, 3101-3110.

12 D. P. Yeggoni, A. Rachamallu and R. Subramanyam, J. Photochem. Photobiol., B, 2016, 160, 248-259.

13 D. P. Yeggoni, A. Rachamallu and R. Subramanyam, $R S C$ Adv., 2016, 6, 40225-40237.

14 D. P. Yeggoni, A. Rachamallu and R. Subramanyam, J. Biomol. Struct. Dyn., 2015, 33, 1336-1351.

15 W.-W. Chao and B.-F. Lin, China's Med., 2010, 5, 1.

16 Y. C. Shen, C. F. Chen and W. F. Chiou, Br. J. Pharmacol., 2002, 135, 399-406.

17 Y.-F. Xia, B.-Q. Ye, Y.-D. Li, J.-G. Wang, X.-J. He, X. Lin, X. Yao, D. Ma, A. Slungaard and R. P. Hebbel, J. Immunol., 2004, 173, 4207-4217.

18 E. Amroyan, E. Gabrielian, A. Panossian, G. Wikman and H. Wagner, Phytomedicine, 1999, 6, 27-31.

19 N. P. Trivedi, U. M. Rawal and B. P. Patel, Integr. Cancer Ther., 2007, 6, 271-280.

20 J. Xu, Z. Li, M. Cao, H. Zhang, J. Sun, J. Zhao, Q. Zhou, Z. Wu and L. Yang, Int. J. Biol. Macromol., 2012, 51, 738-742.

21 K. Vuignier, J. Schappler, J.-L. Veuthey, P.-A. Carrupt and S. Martel, Anal. Bioanal. Chem., 2010, 398, 53-66.

22 J. Koch-Weser and E. M. Sellers, N. Engl. J. Med., 1976, 294, 526-531.

23 J. Ghuman, P. A. Zunszain, I. Petitpas, A. A. Bhattacharya, M. Otagiri and S. Curry, J. Mol. Biol., 2005, 353, 38-52.

24 N. Desai, V. Trieu, Z. Yao, L. Louie, S. Ci, A. Yang, C. Tao, T. De, B. Beals and D. Dykes, Clin. Cancer Res., 2006, 12, 1317-1324.

25 K. Donaldson, V. Stone, C. Tran, W. Kreyling and P. J. Borm, Occup. Environ. Med., 2004, 61, 727-728. 
26 H. Chen, C. Khemtong, X. Yang, X. Chang and J. Gao, Drug Discovery Today, 2011, 16, 354-360.

27 D. B. Shenoy, A. A. Antipov, G. B. Sukhorukov and H. Möhwald, Biomacromolecules, 2003, 4, 265-272.

28 F. Caruso, Adv. Mater., 2001, 13, 11-22.

29 R. Subramanyam, A. Gollapudi, P. Bonigala, M. Chinnaboina and D. G. Amooru, J. Photochem. Photobiol., B, 2009, 94, 8-12.

30 M.-B. Gholivand, A. R. Jalalvand, H. C. Goicoechea and M. Omidi, Spectrochim. Acta, Part A, 2013, 115, 516-527.

31 Å. Frostell-Karlsson, A. Remaeus, H. Roos, K. Andersson, P. Borg, M. Hämäläinen and R. Karlsson, J. Med. Chem., 2000, 43, 1986-1992.

32 D. P. Yeggoni, M. Gokara, D. Mark Manidhar, A. Rachamallu, S. Nakka, C. S. Reddy and R. Subramanyam, Mol. Pharm., 2014, 11, 1117-1131.

33 B. Sudhamalla, M. Gokara, N. Ahalawat, D. G. Amooru and R. Subramanyam, J. Phys. Chem. B, 2010, 114, 9054-9062.

34 T. Mosmann, J. Immunol. Methods, 1983, 65, 55-63.

35 C. Aromdee, Expert Opin. Ther. Pat., 2014, 24, 1129-1138.

36 F. Zsila, Z. Bikádi and M. Simonyi, Biochem. Pharmacol., 2003, 65, 447-456.

37 N. Tayeh, T. Rungassamy and J. R. Albani, J. Pharm. Biomed. Anal., 2009, 50, 107-116.

38 E. Rahnama, M. Mahmoodian-Moghaddam, S. KhorsandAhmadi, M. R. Saberi and J. Chamani, J. Biomol. Struct. Dyn., 2015, 33, 513-533.

39 M. Zolfagharzadeh, M. Pirouzi, A. Asoodeh, M. R. Saberi and J. Chamani, J. Biomol. Struct. Dyn., 2014, 32, 1936-1952.

40 J. R. Lakowicz, Principles offluorescence spectroscopy, Springer, 2009.

41 D. Agudelo, P. Bourassa, J. Bruneau, G. Berube, E. Asselin and H.-A. Tajmir-Riahi, PLoS One, 2012, 7, e43814.

42 S. Tayyab, M. S. Zaroog, S. R. Feroz, S. B. Mohamad and S. N. A. Malek, Int. J. Pharm., 2015, 491, 352-358.
43 M. R. Ajmal, A. S. Abdelhameed, P. Alam and R. H. Khan, Spectrochim. Acta, Part A, 2016, 159, 199-208.

44 J. Min, X. Meng-Xia, Z. Dong, L. Yuan, L. Xiao-Yu and C. Xing, J. Mol. Struct., 2004, 692, 71-80.

45 D. Agudelo, G. Bérubé and H. Tajmir-Riahi, Int. J. Biol. Macromol., 2016, 88, 354-360.

46 S. R. Feroz, S. B. Mohamad, N. Bujang, S. N. Malek and S. Tayyab, J. Agric. Food Chem., 2012, 60, 5899-5908.

47 R. Subramanyam, M. Goud, B. Sudhamalla, E. Reddeem, A. Gollapudi, S. Nellaepalli, V. Yadavalli, M. Chinnaboina and D. G. Amooru, J. Photochem. Photobiol., B, 2009, 95, 81-88.

48 M. Gokara, B. Sudhamalla, D. G. Amooru and R. Subramanyam, PLoS One, 2010, 5, e8834.

49 S. Urien, E. Albengres, J.-L. Pinquier and J.-P. Tillement, Clin. Pharmacol. Ther., 1986, 39, 683-689.

50 D. Morin, N. Simon, P. Depres-Brummer, F. Levi, J.-P. Tillement and S. Urien, Pharmacology, 1997, 54, 271-275. 51 Z. Israili and P. Dayton, Drug Metab. Rev., 2001, 33, 161-235. 52 G. Kumar, U. Walle, K. Bhalla and T. Walle, Res. Commun. Chem. Pathol. Pharmacol., 1993, 80, 337-344.

53 D. N. Bailey and J. R. Briggs, Ther. Drug Monit., 2004, 26, 4043.

54 L. Whitmore and B. A. Wallace, Biopolymers, 2008, 89, 392-400.

55 M. Kallubai, A. Rachamallu, D. P. Yeggoni and R. Subramanyam, Mol. Biosyst., 2015, 11, 1172-1183.

56 M. Gokara, T. Malavath, S. K. Kalangi, P. Reddana and R. Subramanyam, J. Biomol. Struct. Dyn., 2014, 32, 12901302.

57 M. Gokara, G. B. Kimavath, A. R. Podile and R. Subramanyam, J. Biomol. Struct. Dyn., 2015, 33, 196-210.

58 X.-X. Cheng, Y. Lui, B. Zhou, X.-H. Xiao and Y. Liu, Spectrochim. Acta, Part A, 2009, 72, 922-928. 